\title{
O impacto do envelhecimento no paciente hospitalizado: análise do risco nutricional
}

\author{
The impact of aging on hospitalized patients: analysis of nutritional risk
}

\section{DOI: $10.37111 /$ braspenj.2020351011}

Mariana Frigo de Moraes

Jaques Waisberg ${ }^{2}$

Maria de Lourdes do Nascimento da Silva ${ }^{3}$

Fernanda Cristina Alves de Lima ${ }^{4}$

Diogo Oliveira Toledo 5

\section{Unitermos:}

Desnutrição. Idoso. Nutrição de grupos de risco. Morte.

\section{Keywords:}

Malnutrition. Aged. Nutrition for vulnerable groups. Death.

\section{Endereço para correspondência: \\ Mariana Frigo de Moraes}

Rua Agostinho Gomes, 698 - Ipiranga - São Paulo, SP, Brasil - CEP 04206-000.

E-mail: m-frigo@uol.com.br

\section{Submissão}

12 de novembro de 2019

Aceito para publicação 25 de março de 2020

\begin{abstract}
RESUMO
Objetivo: O objetivo deste trabalho foi analisar a presença de risco nutricional e a sua correlação com desfecho clínico, em pacientes idosos hospitalizados. Método: As variáveis do estudo foram peso, altura, índice de massa corporal (IMC), risco nutricional, tempo de internação e óbito dos pacientes internados em um hospital público de São Paulo. Esses dados foram obtidos por meio da Ficha de Atendimento Nutricional, preenchida pelos nutricionistas da instituição. O risco nutricional foi determinado pelo instrumento de triagem nutricional NRS-2002. Para verificar a associação entre presença de risco nutricional e demais variáveis do estudo, utilizou-se teste de Rao \& Scott e regressão logística múltipla (stepwise forward), com nível de significância de $5 \%$. Procedeu-se à análise univariada, sendo que as variáveis com $p<0,20$, em ordem crescente de entrada, foram incluídas na regressão múltipla. Permaneceram, no modelo, as variáveis com $p<0,05$, ou aquelas que ajustaram em, no mínimo, $10 \%$ o valor de odds ratio das demais variáveis. Resultados: Foram avaliadas as fichas de 2613 pacientes, com idade média de 73,87 anos, 54,84\% do sexo feminino, $47,26 \%$ da população estava com risco nutricional e $37,3 \%$ desnutridos. A análise do risco nutricional, segundo os grupos etários evidenciou que, quanto maior a idade, maior a prevalência do risco para a desnutrição. Houve uma boa correlação estatística com o IMC, visto que o risco foi mais prevalente no grupo de desnutridos $(54,41 \%)$. Verificou-se que $11,9 \%$ dos pacientes que possuíam risco nutricional foram a óbito, e $1,66 \%$ dos que não apresentaram risco. Conclusão: $A$ partir deste estudo, foi possível constatar que, para os idosos estudados, o risco para desnutrição se correlacionou de forma positiva com idade, IMC, tempo de internação e ocorrência de óbitos.
\end{abstract}

\section{ABSTRACT}

Objective: The purpose of this study was to analyze the presence of nutritional risk and their correlation with clinical outcome in elderly hospitalized patients. Methods: The study variables were weight, height, body mass index (BMI), nutritional risk, length of stay and death of hospitalized patients in a public hospital in São Paulo. These data were obtained by Sheet Nutritional Care, dietitians completed by the institution. Nutritional risk was determined by nutritional screening tool NRS-2002. To investigate the association between the presence of nutritional risk and other study variables, we used Rao \& Scott test and multiple logistic regression (stepwise forward), with $5 \%$ significance level. It proceeded to univariate analysis, and variables with $p<0.20$, in ascending order of entry were included in multiple regression. They remained in the model the variables with $p<0.05$, or those set by at least $10 \%$ the value of odds ratio of other variables. Results: We evaluated the records of 2613 patients, with a mean age of 73.87 years, $54.84 \%$ female, $47.26 \%$ of the population were at nutritional risk and $37.3 \%$ undernutrition. The nutritional risk groups according to age showed that the greater the age, the prevalence greater risk for malnutrition. There was a good statistical correlation with BMI, because the risk was more prevalent in malnourished group $(54.41 \%)$. It was found that $11.9 \%$ of those who had died nutritional risk, while those who did not risk, only $1.66 \%$. Conclusion: From this study it was found that for older people studied the risk for malnutrition correlated positively with age, BMl, length of stay and the occurrence of deaths.

1. Mestre em Ciências da Saúde, Instituto de Assistência Médica ao Servidor Público Estadual (IAMSPE); Nutricionista coordenadora da área clínica do Hospital do Servidor Público Estadual, São Paulo, SP, Brasil.

2. Doutor em Técnica Operatória e Cirurgia Experimental, Escola Paulista de Medicina da Universidade Federal de São Paulo (UNIFESP); Pósdoutorado e livre docência pela Disciplina de Gastroenterologia Cirúrgica do Departamento de Cirurgia da UNIFESP; Coordenador do Programa de Pós-Graduação Stricto Sensu em Ciências da Saúde do Instituto de Assistência Médica ao Servidor Público Estadual (IAMSPE), Pró-Reitor de Pós-Graduação do IAMSPE, São Paulo, SP, Brasil.

3. Mestre e doutora em Saúde Pública, Faculdade de Saúde Pública da Universidade de São Paulo (USP); Responsável pela Equipe Interdisciplinar de Gerontologia e pelo Aprimoramento em Atendimento Interdisciplinar em Gerontologia e Geriatria do Serviço de Geriatria e Gerontologia do Hospital do Servidor Público Estadual (HSPE); Membro da Comissão de Pós-Graduação, Comissão de Ensino e Pesquisa Multidisciplinar e Comitê de Ética do HSPE, São Paulo, SP, Brasil.

4. Mestre em Ciências da Saúde, Instituto de Assistência Médica ao Servidor Público Estadual (IAMSPE), Nutricionista Cirurgia Geral Hospital do Servidor Público Estadual (HSPE), docente do curso de graduação em Nutrição HOTEC, docente do curso de Pós-Graduação do Centro Universitário São Camilo, São Paulo, SP, Brasil.

5. Mestre em Ciências da Saúde, Instituto de Assistência Médica ao Servidor Público Estadual (IAMSPE), Presidente da BRASPEN, coordenador da equipe multidisciplinar de terapia nutricional (EMTN) dos Hospitais São Luiz Itaim e Albert Einstein, São Paulo, SP, Brasil. 


\section{INTRODUÇÃO}

Nas últimas décadas, no Brasil e no mundo, nota-se um processo sistemático de envelhecimento da população. Atualmente, o Brasil possui mais de 20 milhões de idosos, o que corresponde a $10,8 \%$ da população total, estima-se que no ano de 2050 esse número ultrapasse os 64 milhões, representando mais de $22 \%$ da população'.

Os idosos mais velhos, ou seja, aqueles que possuem 80 anos ou mais, compreendiam aproximadamente 80 milhões de pessoas em 2000, com uma projeção de 395 milhões em 2050, um incremento de 4,9 vezes. Com efeito, apesar de os indivíduos com mais de 80 anos representarem cerca de $1 \%$ da população mundial e 3\% da população em regiões desenvolvidas, esta faixa etária é o segmento da população que cresce mais rapidamente ${ }^{2}$.

O envelhecimento, apesar de ser um processo natural, submete o organismo a diversas alterações anatômicas e funcionais, com repercussões nas condições de saúde e nutrição do idoso. As alterações biológicas próprias da senescência incluem progressiva diminuição da massa corporal magra e de líquidos corpóreos, aumento da quantidade de tecido gorduroso, diminuição de órgãos e acentuada perda de músculos esqueléticos. Todos esses aspectos justificam a busca de condutas e diagnósticos nutricionais que visem à melhora da qualidade de vida desse grupo etário ${ }^{3}$.

Indivíduos acima dos 60 anos passam por mudanças que aumentam as chances de desenvolver desnutrição, a qual constitui um problema comum no envelhecimento, que se origina de uma combinação de fatores ${ }^{4}$.

Assim, a identificação dos pacientes que possam ter seu estado nutricional comprometido durante sua hospitalização torna-se necessária. Porém, é relevante destacar que os instrumentos utilizados para fazer a avaliação nutricional foram previstos para diagnosticar a desnutrição já instalada e não o risco do seu aparecimento, ou seja, o risco nutricional ${ }^{5}$.

Risco nutricional pode ser definido como risco aumentado de morbimortalidade em decorrência de deficiências nutricionais. Este rastreamento evidencia precocemente os pacientes com risco de desnutrição, por meio de medidas rápidas, fáceis e de baixo custo, não sendo necessário estabelecer o diagnóstico nutricional, diferente da avaliação nutricional que utiliza medidas antropométricas, bioquímicas, clínicas e dietéticas ${ }^{5}$.

Não há consenso quanto ao melhor instrumento de triagem nutricional, pois os descritos na literatura possuem limitações, vantagens e desvantagens quando utilizados em populações específicas ${ }^{6}$.

A NRS-2002 é o instrumento de triagem recomendado pela Sociedade Europeia de Nutrição Parenteral e Enteral
(ESPEN) e tem por objetivo detectar o risco de desnutrição no ambiente hospitalar, podendo ser aplicada em adultos, independentemente da idade ${ }^{7}$.

Devido à relevância da detecção precoce do risco nutricional, a fim de se evitar que a desnutrição se instale ou se agrave, este trabalho propõe analisar a presença de risco nutricional e a sua correlação com desfecho clínico em pacientes idosos hospitalizados.

\section{MÉTODO}

presente estudo foi do tipo transversal, aprovado pelo comitê de ética (parecer 227.891 e CAAE: 11184412.7 .0000 .5463 ) para ser desenvolvido nas enfermarias do Hospital do Servidor Público Estadual Francisco Morato (HSPE-FMO) - clínica médica, cirurgia geral, geriatria, moléstias infecciosas, cirurgia vascular, urologia, oncologia, hematologia, neuroclínica, neurocirurgia, gastroclínica, gastrocirurgia, doenças do aparelho respiratório, ortopedia, cardiologia, ginecologia, cirurgia de cabeça e pescoço.

protocolo de atendimento nutricional do HSPEFMO prevê que todos os pacientes devem ser triados para avaliação do risco nutricional pelos nutricionistas em até $48 \mathrm{~h}$ da sua admissão hospitalar, todas as informações obtidas nesta avaliação são registradas na Ficha de Atendimento Nutricional e por meio desta ficha foram obtidos os dados necessários para a realização desse trabalho [idade, peso, altura, índice de massa corporal (IMC), resultado da triagem de risco nutricional].

O tempo de internação hospitalar corresponde ao período em que cada paciente do estudo foi admitido no hospital até a data da sua alta ou óbito. As informações da data de internação e de alta hospitalar ou óbito, necessárias para calcular o tempo de internação, foram obtidas por meio do programa interno do hospital chamado Sistema de Informação e Gestão Hospitalar (SIGH).

Foram avaliadas, num período de três meses, as fichas de Atendimento Nutricional de 2613 pacientes idosos, de ambos os gêneros, internados nas enfermarias do hospital, tendo como variáveis: idade; IMC segundo a Organização Pan-Americana da Saúde (OPAS), risco nutricional (triagem nutricional NRS-2002); tempo de internação e óbito.

Para aferir o peso corporal dos pacientes no momento da admissão hospitalar, utilizou-se balança digital Filizola ${ }^{\circledR}$, já a estatura foi aferida com fita métrica de precisão de até décimos de centímetros ( $\mathrm{mm}$ ), afixada na parede lisa e sem rodapé. Nos pacientes acamados, foram aplicadas as fórmulas de Chumlea para a estimativa de peso e de altura. 
O risco nutricional é definido através do questionário de triagem nutricional NRS-2002, o qual é composto por 2 partes. A primeira parte contém perguntas referentes ao IMC do paciente entrevistado, se ele apresentou perda de peso nos últimos 3 meses, se houve diminuição da ingestão alimentar na última semana e se ele está com mal estado geral. Se alguma dessas questões tiver resposta positiva, a investigação será prosseguida para a segunda parte do questionário, a qual contém perguntas sobre o estado nutricional e a gravidade da doença. Após o preenchimento do questionário, é determinada a presença de risco nutricional por meio da pontuação total: pacientes que apresentarem na avaliação pontuação final $\mathrm{P} \geq 3$ : risco nutricional e pontuação final $\mathrm{P}<$ 3: sem risco no nutricional.

A descrição da população, segundo variáveis de estudo, foi apresentada por meio de frequência absoluta, relativa, valores médios e desvio padrão, com nível de significância de 5\%.

Para verificar a associação entre presença de risco nutricional e demais variáveis de estudo, utilizou-se teste de Rao \& Scott e regressão logística múltipla (stepwise forward), com nível de significância de 5\%.

Procedeu-se à análise univariada, sendo que as variáveis com $p<0,20$, em ordem crescente de entrada, foram incluídas na regressão múltipla.

Permaneceram, no modelo, as variáveis com $p<0,05$, ou aquelas que ajustaram em, no mínimo, 10\% o valor de odds ratio das demais variáveis.

Foi utilizado o Excel para digitação dos dados e o programa estatístico STATA/SE 10.1 para realização dos cálculos.

\section{RESULTADOS}

A população foi composta por 2613 idosos hospitalizados, sendo a maioria $(54,84 \%)$ do gênero feminino.

A idade média dos indivíduos foi de 73,87 $\pm 0,17$ anos, variando de 60 a 106 anos. $\bigcirc$ tempo médio de internação foi de 12,13 $\pm 0,25$ dias, com tempo máximo de internação de 135 dias, sendo óbito o desfecho clínico de 6,51\% dos indivíduos avaliados.

A ferramenta de triagem nutricional NRS-2002 demonstrou que $47,26 \%$ dos pacientes apresentavam risco nutricional. Segundo o IMC, 37,3\% dos pacientes estavam com o estado nutricional adequado, contudo $34,4 \%$ dos indivíduos estavam desnutridos, sendo a média do IMC de $25,42 \pm 0,10 \mathrm{~kg} / \mathrm{m}^{2}$. A descrição completa da população é apresentada na Tabela 1.

Avaliando o risco nutricional, foi possível verificar que esta variável apresentou diferença significativa entre os gêneros, com maior prevalência no sexo feminino (52,39\%). Segundo
Tabela 1 - Caracterização de idosos hospitalizados, segundo variáveis de estudo.

\begin{tabular}{|c|c|}
\hline $\begin{array}{l}\text { Variáveis de } \\
\text { estudo }\end{array}$ & $\begin{array}{c}\text { Idosos hospitalizados } \\
(n=2.613)\end{array}$ \\
\hline Idade $\mathrm{M}( \pm \mathrm{DP})$ & $73,87(0,17)$ \\
\hline Mulheres (\%) & $54,84(\mathrm{~N}=1.433)$ \\
\hline Tempo de internação M ( \pm DP) & $12,13(0,25)$ \\
\hline Óbito (\%) & $6,51(\mathrm{~N}=170)$ \\
\hline Presença de risco nutricional (\%) & $47,26(\mathrm{~N}=1.235)$ \\
\hline $\mathrm{IMC} \mathrm{M}( \pm \mathrm{DP})$ & $25,42(0,10)$ \\
\hline \multicolumn{2}{|l|}{ Estado nutricional - IMC/OPAS (\%) } \\
\hline Peso adequado & $37,31(\mathrm{~N}=975)$ \\
\hline Desnutrição & $34,40(\mathrm{~N}=899)$ \\
\hline Risco para obesidade & $9,45(\mathrm{~N}=247)$ \\
\hline Obesidade & $18,83(\mathrm{~N}=492)$ \\
\hline
\end{tabular}

FONTE: Ficha de Atendimento Nutricional SND/HSPE-FMO, 2012. Nota: Informações em valores médios (M), desvio padrão (DP \pm ), frequência absoluta (N) e relativa (\%), com nível de significância de 5\%.

os grupos etários evidenciou que, quanto maior a idade, maior a prevalência do risco para a desnutrição, no grupo com idade entre 70 a 79 anos, o risco nutricional foi de $42,67 \%$ e, no grupo com idade de $\geq 80$ anos, $40 \%$. Houve boa correlação entre o risco nutricional e o IMC, pois o risco foi mais prevalente no grupo de desnutridos $(54,41 \%)$.

Verificando a relação do risco nutricional com o tempo de internação, foi possível constatar que os pacientes que permaneceram internados durante o período de 8 a 15 dias apresentaram maior prevalência de risco nutricional.

Em relação ao desfecho hospitalar, verificou-se que 11,9\% dos pacientes que possuíam risco nutricional foram a óbito, enquanto os que não apresentaram risco, apenas $1,66 \%$. A descrição completa das variáveis segue na Tabela 2 .

A partir da análise univariada, foi possível contatar que: os idosos do sexo masculino $(O R=1,21 ; I C=1,03-1,41)$, dos grupos etários 70 -79 $(\mathrm{OR}=3,94 ; \mathrm{IC}=3,23-4,80)$ e $\geq 80$ anos $(\mathrm{OR}=7,24 ; \mathrm{IC}=5,81-9,02)$, com tempo de internação de 8 a 15 dias $(O R=2,37 ; I C=1,97-2,85), 16$ a 22 dias $(O R=3,04$; $\mathrm{IC}=2,34-3,95)$, e $>22$ dias $(\mathrm{OR}=5,06 ; \mathrm{IC}=3,89-6,58)$, que foram ao óbito $(O R=7,96 ; I C=5,09-12,44)$ e que foram classificados como desnutridos pelo IMC $(O R=4,56$; $I C=3,74-5,55)$, apresentaram maiores chances para risco nutricional hospitalar, com diferença estatística (Tabela 3).

Dentre as variáveis avaliadas, as que demonstraram maior chance de ter risco nutricional foram: os idosos que foram a óbito (5,29 vezes); grupos etários 70-79 anos (4,29 vezes); $\geq 80$ anos (7,21 vezes); tempo de internação de 8 a 15 dias $(2,29$ vezes); 16 a 22 dias $(2,86$ vezes); $>22$ dias $(4,43$ vezes); sexo masculino (1,19 vezes), com nível de significância $\mathrm{p}<0,05$ (Tabela 4). 
Tabela 2 - Prevalência de risco nutricional em idosos hospitalizados, estimado pela NRS 2002, segundo variáveis de estudo.

\begin{tabular}{|c|c|c|c|}
\hline \multicolumn{4}{|c|}{ Prevalência de risco nutricional (NRS 2002) } \\
\hline Variáveis de estudo & $\begin{array}{c}\text { Não } \\
\% \text { (N) }\end{array}$ & $\begin{array}{l}\text { Sim } \\
\%(\mathrm{~N})\end{array}$ & Valor de $p$ \\
\hline \multicolumn{4}{|l|}{ Sexo } \\
\hline Homem & $42,96(786)$ & $47,61(647)$ & 0,017 \\
\hline Mulher & $57,04(592$ & $52,39(588)$ & \\
\hline \multicolumn{4}{|l|}{ Grupos etários } \\
\hline 70-79 anos & $32,15(443)$ & $42,67(527)$ & 0,000 \\
\hline 80 anos e mais & $16,40(226)$ & $40,00(494)$ & \\
\hline \multicolumn{4}{|l|}{ Tempo de internação } \\
\hline 0-7 dias & $56,89(784)$ & $30,85(381)$ & \\
\hline 8-15 dias & $27,07(373)$ & $34,74(429)$ & 0,000 \\
\hline \multicolumn{4}{|l|}{ Óbito } \\
\hline Não & $98,33(1.355)$ & $88,10(1.088)$ & 0,000 \\
\hline Sim & $1,67(23)$ & $11,90(147)$ & \\
\hline \multicolumn{4}{|c|}{ Estado Nutricional - IMC/OPAS 2002} \\
\hline Peso adequado & $42,98(591)$ & $31,09(384)$ & \\
\hline Desnutrição & 27,21 (227) & $54,41(672)$ & 0,000 \\
\hline Risco para obesidade & $13,43(185)$ & $5,02(62)$ & \\
\hline Obesidade & $16,47(375)$ & 9,47 (117) & \\
\hline
\end{tabular}

FONTE: Ficha de Atendimento Nutricional SND/HSPE-FMO, 2012.

Teste de Rao \& Scott e regressão logística múltipla (stepwise forward), com nível de significância de 5\%.

Tabela 3 - Análise univariada de associação entre risco nutricional de idosos hospitalizados, estimado pela NRS 2002 e variáveis de estudo.

\begin{tabular}{|c|c|c|c|}
\hline Variáveis de estudo & OR & IC (95\%) & Valor de $p$ \\
\hline \multicolumn{4}{|l|}{ Sexo } \\
\hline Homem & 1 & & \\
\hline \multicolumn{4}{|l|}{ Grupos etários } \\
\hline $60-69$ anos & 1 & & \\
\hline 80 anos e mais & 7,24 & $5,81-9,02$ & \\
\hline \multicolumn{4}{|l|}{ Tempo de internação } \\
\hline 0-7 dias & 1 & & \\
\hline $8-15$ dias & 2,37 & $1,97-2,85$ & \\
\hline $16-22$ dias & 3,04 & $2,34-3,95$ & 0,000 \\
\hline Sim & 7,96 & $5,09-12,44$ & \\
\hline \multicolumn{4}{|c|}{ Estado Nutricional - IMC/OPAS 2002} \\
\hline Peso adequado & 1,00 & & \\
\hline Desnutrição & 4,56 & $3,74-5,55$ & 0,000 \\
\hline Risco para obesidade & 0,52 & $0,38-0,71$ & \\
\hline Obesidade & 0,48 & $0,38-0,61$ & \\
\hline
\end{tabular}




\begin{tabular}{|c|c|c|c|c|}
\hline Variáveis de estudo & OR & IC (95\%) & $P$ da variável & $\mathrm{P}$ do modelo \\
\hline \multicolumn{5}{|l|}{ Óbito } \\
\hline Não & 1 & & & \\
\hline Sim & 5,29 & $3,27-8,51$ & 0,000 & \\
\hline \multicolumn{5}{|l|}{ Grupos etários } \\
\hline $60-69$ anos & 1 & & & \\
\hline $70-79$ anos & 4,29 & $3,47-5,30$ & 0,000 & \\
\hline 80 anos e mais & 7,21 & $5,71-9,10$ & 0,000 & \\
\hline Tempo de internação & & & & 0,000 \\
\hline $0-7$ dias & 1 & & & \\
\hline $8-15$ dias & 2,29 & $1,88-2,81$ & 0,000 & \\
\hline $16-22$ dias & 2,86 & $2,15-3,82$ & 0,000 & \\
\hline$>22$ dias & 4,43 & $3,31-5,94$ & 0,000 & \\
\hline \multicolumn{5}{|l|}{ Sexo } \\
\hline Mulher & 1 & & & \\
\hline Homem & 1,19 & $1,00-1,41$ & 0,05 & \\
\hline
\end{tabular}

FONTE: Ficha de Atendimento Nutricional SND/HSPE-FMO, 2012.

Nota: OR - odds ratio; IC - intervalo de confiança; ${ }^{*} p<0,05$. Regressão logística múltipla.

\section{DISCUSSÃO}

No presente estudo, os pacientes com 70 anos ou mais constituíram a maioria dos indivíduos, com prevalência do gênero feminino. Segundo o Fundo de População das Nações Unidas (UNFPA), há predominância do sexo feminino entre os idosos. Em 2010, as mulheres representavam 10,5\% da população idosa total, enquanto os homens $8,4 \%$ e a projeção para 2020 é de $14 \%$, enquanto os homens apenas $11 \%$.

O tempo médio de internação dos idosos avaliados no presente trabalho foi semelhante ao de outros estudos da literatura. $\mathrm{O}$ estudo de Bretón et al. ${ }^{9}$ avaliou, por meio do Mini Nutritional Assessment (MNA) e do Nutritional Risk Screening (NRS)-2002, a prevalência de risco para desnutrição em pacientes hospitalizados e sua capacidade de prever complicações clínicas. Foram avaliados 57 pacientes adultos e idosos, com idade média de 70, 5 anos e o tempo médio de internação foi de 9,56 dias.

Em 2012, Álvarez-Hernández et al. ${ }^{10}$ conduziram o estudo PREDyCES ${ }^{\circledR}$, que teve como objetivos principais analisar a prevalência do risco nutricional na Espanha, tanto na admissão quanto na alta hospitalar, e estimar os custos hospitalares associados à desnutrição. Foi um projeto multicêntrico, que envolveu 31 hospitais, 1707 pacientes adultos e idosos. O risco nutricional foi avaliado por meio da NRS-2002. A idade média da população do estudo foi 63 anos, $55 \%$ tinham $\geq 65$ anos e o tempo médio de internação foi de 8,9 dias $^{10}$.

López et al. ${ }^{11}$ estudaram a prevalência do risco nutricional em 277 pacientes adultos e idosos, com idade média de 67,71 anos. Em $62,81 \%$ da população, a idade foi $\geq 65$ anos e o tempo médio de internação 9,1 dias. presente estudo teve como um dos seus objetivos conhecer o número de óbitos da população estudada durante o período da pesquisa. Com a finalidade de identificar fatores que se relacionavam a piores desfechos clínicos (maior tempo de internação e óbito) em pacientes idosos hospitalizados, De Buyser et al. ${ }^{12}$ avaliaram 1123 indivíduos, com idade média de 81,5 anos, $56 \%$ eram mulheres. $\bigcirc$ tempo médio de internação foi de 10 dias e o óbito ocorreu em $4 \%$ da população, dados semelhantes ao atual estudo.

Já López-Gómez et al. ${ }^{13}$, no estudo para avaliar o rastreio de risco nutricional, tempo de internação e mortalidade hospitalar, avaliaram 113 pacientes, com idade média de 80,86 anos, e a mortalidade foi de $14 \%$. Esse valor é maior do que o encontrado no presente trabalho, provavelmente devido à média de idade da população também ter sido maior e quanto maior a idade, maior a chance de óbitos dos indivíduos ${ }^{13}$.

No presente estudo, o rastreamento realizado com a NRS-202 resultou em quase a metade dos pacientes com risco nutricional. Esta porcentagem salienta a importância do rastreamento nutricional para identificar os indivíduos que estão mal nutridos ou em risco de desenvolver desnutrição ${ }^{5}$.

Diversos estudos buscam divulgar a prevalência do risco para a desnutrição no ambiente hospitalar e para isso utilizam o instrumento NRS-2002, visto que o seu uso foi recomendado pela ESPEN, os valores encontrados variam de $20 \%$ a $60 \%$.

Mercadal-Orfila et al. ${ }^{14}$ avaliaram o risco nutricional rastreado pela NRS-2002 e sua relação com a morbimortalidade de pacientes hospitalizados. Foram avaliados 1075 
indivíduos, com idade média de 67,9 anos, 62\% da população apresentaram risco nutricional ${ }^{14}$.

Em 2013, foi realizada uma pesquisa com a finalidade de testar a validade de ferramentas de triagem nutricional [Malnutrition Universal Screening Tool (MUST), MNA e NRS-2002], em pacientes idosos hospitalizados, sua associação com risco nutricional e mortalidade. A pesquisa envolveu 233 pacientes, com idade média de 81 anos, o risco nutricional foi verificado em $54 \%$ da população ${ }^{15}$.

Alvarez-Altamirano et al. ${ }^{16}$ tiveram como objetivo aplicar a NRS-2002 para determinar a prevalência e fatores associados ao risco nutricional. Foram avaliados 444 pacientes adultos e idosos, dos quais $50 \%$ apresentaram risco nutricional.

Pode-se verificar que os resultados deste trabalho são compatíveis com os de outros pesquisadores, revelando a importância de se estabelecer protocolos de atendimento nutricional que incluam o rastreio do risco para a desnutrição, a fim de otimizar a identificação dos pacientes que irão se beneficiar de uma intervenção nutricional precoce e adequada. O IMC é um indicador do estado nutricional, a média encontrada na atual pesquisa foi de eutrofia, caracterizando estado nutricional adequado. Contudo, apesar dessa média de IMC, 34,4\% dos indivíduos avaliados foram classificados como desnutridos, ou seja, IMC menor $23 \mathrm{~kg} / \mathrm{m}^{2}$.

Em um estudo realizado por Cintra et al. ${ }^{17}$, no qual foram estudados 304 pacientes internados, de ambos os sexos, a prevalência de $20 \%$ de desnutrição, sendo a população idosa a mais atingida ${ }^{17}$. Em 2013, no estudo BRAINS ${ }^{18}$, a desnutrição foi detectada em $30,8 \%$ da população de idosos avaliada.

É importante destacar que do total de pacientes em risco nutricional ( $n=1235), 889$ já estavam desnutridos, de acordo com o IMC. Assim, 28,01\% dos idosos com risco nutricional podiam ainda não apresentar desnutrição à admissão, demonstrando a importância da adoção de medidas preventivas e eficazes para evitar que a mesma se desenvolva durante a permanência hospitalar.

Em pacientes hospitalizados, a desnutrição é causada pela combinação de diversos fatores inerentes à condição do paciente e ao tratamento, as quais ocorrem antes, durante e após sua internação, tais como a doença de base, comorbidades agudas e crônicas, efeitos colaterais de medicamentos, inatividade física, além de negligência das equipes de saúde quanto aos aspectos nutricionais em detrimento de outros cuidados $^{19}$.

É correto afirmar que a desnutrição pode se desenvolver durante o período de internação hospitalar, em decorrência da própria patologia e do seu tratamento, bem como por fatores ligados à alimentação. Jejum prolongado para realização de exames e cirurgias, dietas restritivas e baixa aceitação alimentar também contribuem de forma marcante para que a desnutrição se instale ou se agrave.

Em 2009, ocorreu no Brasil a primeira edição do NutriDia, que é um projeto multicêntrico vinculado ao Nutrition Day, iniciado na Europa em 2006. Sua ideia principal é avaliar, em um único dia, uma ou várias unidades de um mesmo hospital, tentando evidenciar os problemas nutricionais que seus pacientes possuem. A pesquisa foi feita pela SBNPE, com 842 pacientes, 25 hospitais públicos e privados e teve como objetivo a conscientização de profissionais da saúde e instituições médicas sobre a importância dos cuidados nutricionais adequados para a recuperação do paciente ${ }^{20}$.

Os principais achados do NutriDia foram 72 dos pacientes não ingeriam por completo as 3 principais refeições do dia, sendo que $54,8 \%$ não ingeriam todo o almoço e $29,2 \%$ menos da metade. A maioria aceitava melhor o café da manhã. Entre as razões para essa baixa aceitação está a falta de apetite, repulsa pelo sabor, hábito de comer pouco, realização de exames/cirurgias no horário da refeição e náuseas e vômitos ${ }^{20}$.

A dieta hospitalar é importante por garantir o aporte de nutrientes ao paciente internado e, assim, preservar seu estado nutricional. A dicotomia entre dieta e comida manifesta a ruptura entre o prazer, o gosto e o aporte nutricional, de modo a priorizar o atendimento às demandas biológicas ${ }^{21}$.

Todos esses fatores são agravados com a idade, pois o ambiente hospitalar é considerado de grande risco, especialmente para esta população, pois, associado às comorbidades e alterações próprias da faixa etária, contribui fortemente para a instalação do quadro de desnutrição ${ }^{22}$. Este fato pode ser constatado no presente estudo; de acordo com a análise univariada, os idosos dos grupos etários 70-79 e $\geq 80$ anos apresentaram maiores chances para risco nutricional hospitalar.

O estudo PREDyCES ${ }^{10}{ }^{10}$ demonstrou que $23 \%$ da população da Espanha estavam em risco nutricional, porém especificamente em idosos este número se elevou para $37 \%$. Foi possível verificar neste estudo que houve boa correlação entre o risco nutricional e tempo de internação.

Houve correlação positiva entre o óbito e o risco nutricional no presente estudo. O EuroOOPS Study Group realizou um estudo multicêntrico, envolvendo 12 países da Europa, totalizando 26 hospitais. Foram avaliados 5051 pacientes, dos quais $32,6 \%$ apresentaram risco nutricional e a ocorrência de óbito foi mais frequente no grupo de risco nutricional (12\%) quando comparado ao grupo sem risco $(1 \%)^{23}$.

Em 2013, um estudo transversal teve como objetivo avaliar a prevalência de risco nutricional e o tempo de internação de pacientes internados em um Hospital Escola no Rio 
Grande do Sul, do total de indivíduos avaliados, 36,28\% eram idosos ( $\geq 60$ anos) e a maioria do gênero feminino (51\%). Ao observar o desfecho hospitalar dos pacientes, notou-se o aumento de mortalidade conforme o aumento do risco nutricional, zero por cento nos pacientes com baixo risco, passando para $4 \%$ naqueles com médio risco e atingindo o patamar de $13 \%$ nos pacientes com alto risco nutricional $\left(p<0,001\right.$, teste do $\left.\chi^{2}\right)$. Assim, ter risco nutricional alto trouxe uma probabilidade três vezes maior de óbito em relação aos pacientes com médio risco nutricional ${ }^{24}$.

Um estudo prospectivo realizado em seis países da Europa teve como objetivo relacionar a perda de peso com a mortalidade em idosos. Foram selecionados 34.239 participantes e a conclusão do estudo foi que a perda de peso nos idosos está associada com o aumento da mortalidade ${ }^{25}$.

Sabe-se, também, que o risco nutricional se correlaciona com a gravidade da doença, pois quanto mais grave for a condição clínica do paciente, também será maior o seu risco nutricional. $\bigcirc$ estado nutricional preservado é um fator determinante para o adequado funcionamento do sistema imunológico, propiciando o sucesso no tratamento clínico e cirúrgico do paciente. Por isso, a desnutrição aumenta as chances de infecção e complicações e, consequentemente, eleva o tempo de hospitalização e o número de óbitos, o que pode ser verificado por meio dos resultados apresentados no presente trabalho e em diversos outros estudos.

Visto que, quanto maior a idade do paciente, maior o risco nutricional e estes se correlacionam positivamente com maior tempo de internação e ocorrência de óbitos, torna-se relevante a assistência integral ao paciente idoso desde o momento da admissão hospitalar.

\section{CONCLUSÃO}

A partir deste estudo, foi possível constatar que, para os idosos estudados, o risco para desnutrição se correlacionou de forma positiva com idade, IMC, tempo de internação e ocorrência de óbitos.

\section{REFERÊNCIAS}

1. IBGE - Instituto Brasileiro de Geografia e Estatística. Estimativa da população residente para os municípios e para as unidades da federação brasileiros. Rio de Janeiro: Instituto Brasileiro de Geografia e Estatística; 2019.

2. Brasil. Secretaria de Direitos Humanos da Presidência da República. Envelhecimento no Brasil. Brasília: Secretaria de Direitos Humanos da Presidência da República; 2015. [cited 2019 Oct 5]. Available from: http://sdh.gov.br/assuntos/pessoa-idosa/ dados-estatisticos/dados-sbre-o-envelheciemnto-no-brasil.

3. Silva SCM, Aires CN, Figueira YLV, Bogéa MRJ, Mendonça MJ. Alterações fisiológicas do idoso e seu impacto na ingestão alimentar: uma revisão de literatura. Rev Eletr Acervo Saúde. 2017;6:288-95.
4. Cesari M, Marzetti E, Thiem U, Perez-Zepeda MU, Abellan Van Kan G, Landi F, et al. The geriatric management of frailty as paradigm of "The end of the disease era". Eur J Intern Med. 2016;31:11-4.

5. Mueller C, Compher C, Ellen DM; American Society for Parenteral and Enteral Nutrition (A.S.P.E.N.) Board of Directors. A.S.P.E.N. clinical guidelines: nutrition screening, assessment, and intervention in adults. JPEN J Parenter Enteral Nutr. 2011;35(1):16-24.

6. Cederholm T, Jensen GL, Correia MITD, Gonzalez MC, Fukushima R, Higashiguchi T, et al. GLIM criteria for the diagnosis of malnutrition: a consensus report from the global clinical nutrition community. Clin Nutr. 2019;38(1):1-9.

7. Kondrup J, Rasmussen HH, Hamberg O, Stanga Z; ad hoc ESPEN Working Group. Nutritional risk screening (NRS 2002): a new method based on an analysis of controlled clinical trials. Clin Nutr. 2003;22(3):321-36.

8. UNFPA. Fundo de população das Nações Unidas. ONU(2015). Disponível em: http://www.nacoesunidas.org.

9. Bretón MJOJ, Trallero JA, Martinez ABM, Diaz LS, Gutierrez EA, Orna JAG. Comparación de dos herramientas de cribado nutricional para predecir la aparición de complicaciones en pacientes hospitalizados. Nutr Hosp. 2012;27(3):701-6.

10. Álvarez-Hernández J, Planas Vila M, León-Sanz M, García de Lorenzo A, Celaya-Pérez S, García-Lorda P, et al. Prevalence and costs of malnutrition in hospitalized patients: the PREDyCES $\mathbb{R}$ study. Nutr Hosp. 2012;27(4):1049-59.

11. López MTF, Baamil OF, Doldán CL, Alonso MLB, Prada MTS, Labrador FL, et al. Prevalencia de desnutrición en pacientes hospitalizados no críticos. Nutr Hosp. 2014;30(6):1375-83.

12. De Buyser SL, Petrovic M, Taes YE, Vetrano DL, Onder G. A multicomponent approach to identify predictors of hospital outcomes in older in-patients: a multicentre, observational study. PLoS One. 2014; 9(12): e115413. doi:10.1371/journal. pone. 0115413

13. López-Gómez JJ, Calleja-Fernández A, Ballesteros-Pomar MD, Vidal-Casariego A, Brea-Laranjo C, Fariza-Vicente E, et al. Screening of the nutritional risk in elderly hospitalized patients with different tools. Endocrinol Nutr. 2011;58(3):104-11.

14. Mercadal-Orfila G, Lluch-Taltavull J, Campillo-Artero C, Torrent-Quetglas M. Association between nutritional risk based on the NRS-2002 test and hospital morbidity and mortality. Nutr Hosp. 2012;27(4):1248-54.

15. Holst M, Yifter-Lindgren E, Surowiak M, Nielsen K, Mowe M, Carlsson $\mathrm{M}$, et al. Nutritional screening and risk factors in elderly hospitalized patients: association to clinical outcome? Scand J Caring Sci. 2013;27(4):953-61.

16. Alvarez-Altamirano K, Delgadillo T, García-García A, AlatristeOrtiz G, Fuchs-Tarlovsky V. Prevalencia de riesgo de desnutrición evaluada con NRS-2002 en población oncológica mexicana. Nutr Hosp. 2014;30(1):173-8.

17. Cintra RMGC, Garla P, Bosio MC, Tognoli M, Soares AS, Matto MSR, et al. Estado nutricional de pacientes hospitalizados e sua associação com o grau de estresse das enfermidades. Rev Simbio-Logias. 2008;1(1):145-56.

18. Borghi R, Meale MMS, Gouveia MAP, França JID, Damião AOMC. Perfil nutricional de pacientes internados no Brasil: análise de 19.222 pacientes (Estudo BRAINS). Rev Bras Nutr Clin. 2013;28(4):255-63.

19. Beghetto MG. Estado nutricional como preditor de morte, infecção e permanência hospitalar [Tese de Doutorado]. Porto Alegre: Universidade Federal do Rio Grande do Sul; 2007.

20. SBNPE - Sociedade Brasileira de Nutrição Parenteral e Enteral. Projeto NutriDia. [cited 2019 Oct 2]. Available from: http:// www.sbnpe.com.br.

21. Garcia RWD. A dieta hospitalar na perspectiva dos sujeitos envolvidos em sua produção e em seu planejamento. Rev Nutr. 2006;19(2):129-44. 
22. Miranda RNA, Salgado NA, Almeida TTG, Silva TCS, Maciel AP. Métodos de avaliação para a detecção de Desnutrição em idosos Hospitalizados atendidos pelo Programa de Residência Multiprofissional em Saúde do Idoso, Belém/PA. XXII Congresso Brasileiro de Nutrição e III Congresso Ibero-Americano de Nutrição, 2012.

23. Sorensen J, Kondrup J, Prokopowicz J, Schiesser M, Krahenbuhl L, Meier R, et al. EuroOOPS: an international multicentre study to implement nutritional risk screening and evaluate clinical outcome. Clin Nutr. 2008;27(3):340-9.
24. Garcia RS, Tavares LRC, Pastore CA. Rastreamento nutricional em pacientes cirúrgicos de um hospital universitário do sul do Brasil: o impacto do risco nutricional em desfechos clínico. Einstein. 2013;11(2):147-52.

25. Bamia C, Halkjær J, Lagiou P, Trichopoulos D, Tjønneland A, Berentzen TL, et al. Weight change in later life and risk of death amongst the elderly: the European Prospective Investigation into Cancer and Nutrition-Elderly Network on Ageing and Health study. J Intern Med. 2010;268:133-44.

Local de realização do estudo: Hospital do Servidor Público Estadual, São Paulo, SP, Brasil.

Conflito de interesse: Os autores declaram não haver. 